\title{
Estudo parasitológico de amostras fecais encontradas em calçadas no município de Niterói, $\mathbf{R} \mathbf{J}^{*}$
}

\section{Parasitological study of fecal samples found on the sidewalks in Niterói, RJ}

\author{
Luciano Antunes Barros, ${ }^{* *}$ Cathia Maria Barrientos Serra, ${ }^{* *}$ Dalton Garcia de Mattos Júnior, ${ }^{* *}$ Beatriz Coronato Nunes, ${ }^{* * *}$ \\ Leonardo Varon Gaze, ${ }^{* *}$ Mariana Braga Cavalcanti, ${ }^{* *}$ Gustavo Dornelles Machado, ${ }^{* *}$ Daniel Lange de Luna**
}

\begin{abstract}
Resumo
A contaminação ambiental por fezes de cães e gatos tem sido uma preocupação constante em áreas urbanas, devido principalmente à possibilidade de transmissão de zoonoses. É comum o encontro de massas fecais nas calçadas de alguns bairros do município de Niterói. O objetivo deste trabalho foi determinar a prevalência de parasitos em massas fecais recolhidas em oito bairros do município de Niterói. Duzentas e quarenta amostras de massas fecais foram recolhidas em vias públicas e processadas no Laboratório de Doenças Parasitárias da Faculdade de Veterinária da UFF, utilizando-se as técnicas de sedimentação simples e centrifugoflutuação em solução de sulfato de zinco. A prevalência das massas fecais positivas para a presença de formas parasitárias foi de 18,3\%. Destas, 22,7\% foram encontradas em Charitas, 20,7\% em Jurujuba, 13,6\% no Vital Brazil, 13,6\% no Ingá, 11,4\% em São Francisco, 9,1\% em Santa Rosa, 4,5\% em Icaraí e 4,5\% em Piratininga. Das formas parasitárias encontradas, $76 \%$ foram ovos de ancilostomatídeos, $9 \%$ cápsulas ovígeras de Dipylidium sp., $9 \%$ ovos de Trichuris $\mathrm{sp}$., $4 \%$ ovos de Toxocara sp. e $2 \%$ de formas vegetativas de Entamoeba sp. Estes dados são úteis como indicadores de risco para a saúde animal e humana, bem como para campanhas de conscientização com enfoque em Saúde Pública.
\end{abstract}

Paravras-chave: cão, fezes, zoonoses.

\begin{abstract}
The environmental contamination by excrements of dogs and cats has been a constant concern in urban areas, mainly due to the possibility of transmission of zoonosis. It is common to find fecal masses on the sidewalks of some Niterói's boroughs. The objective of this work was to determine prevalence of parasitic agents in collected fecal masses in the public ways of eight quarters of the city of Niterói. Two hundred and forty samples of fecal masses had been collected and processed at the Laboratory of Parasites Diseases at the UFF, by techniques of spontaneous sedimentation and centrifuge-flotation. The prevalence of positive fecal masses for the presence of parasitic forms was of $18,3 \%$. Of these $22,7 \%$ were found in Charitas, $20,4 \%$ in Jurujuba, $13,6 \%$ in Vital Brazil, $13,6 \%$ in Ingá, $11,4 \%$ in São Francisco, 9,1\% in Santa Rosa, 4,5\% in Icaraí and 4,5\% in Piratininga. Of the parasitic forms found $76 \%$ were eggs of ancylostomatids, $9 \%$ egg capsules of Dipylidium sp., $9 \%$ eggs of Trichuris sp., $4 \%$ eggs of Toxocara sp. and $2 \%$ of vegetative forms of Entamoeba sp. Data on the prevalence of parasitic agents in excrements of dogs, are useful as indicator of risk for the animal and human population and in campaigns of awareness about public health.
\end{abstract}

Keywords: dog, feces, zoonosis.

\section{Introdução}

A contaminação ambiental por fezes de cães e gatos tem sido uma preocupação constante em áreas urbanas, devido principalmente à possibilidade de transmissão de zoonoses como larva migrans cutânea, larva migrans visceral, toxoplasmose, criptosporidíase e giardíase. A presença de fezes destes animais em ambiente urbano constitui um problema sanitário relevante para crianças, pois são particularmente mais sujeitas aos riscos de infecção, principalmente na faixa etária de 1 a 6 anos, quando apresentam maior tendência à exploração do ambiente e pouca ou nenhuma noção sobre hábitos de higiene (Acha e Szyfres, 2003).
As áreas de recreação infantil, calçadas e praias são locais onde há grande probabilidade da presença de formas infectantes de agentes zoonóticos. Isto se deve principalmente à falta de conscientização dos proprietários de cães que, associado à ausência de um controle parasitário adequado destes animais, contribui negativamente para a manutenção de vias públicas como áreas livres de risco de infecção.

É comum encontrar massas fecais nas calçadas e praças da área urbana do município de Niterói, principalmente de bairros como Icaraí, Santa Rosa, Vital Brazil, Ingá e São Francisco, onde a verticalização das construções de moradia urbana tem aumentado muito na última década, resultando também em

\footnotetext{
${ }^{*}$ Recebido em 11 de outubro de 2012 e aceito em 13 de abril de 2013.

** Laboratório de Doenças Parasitárias. Faculdade de Veterinária da Universidade Federal Fluminense. Rua Vital Brazil Filho, 64, 24230-340, Santa Rosa, Niterói, RJ. E-mail: lucianobarrosrj@gmail.com

*** Laboratório de Parasitologia. Instituto Biomédico da Universidade Federal Fluminense. Rua prof. Hermani de Mello, 101, sala 212C. São Domingos, 24210-130. Niterói, RJ.
} 
aumento do número de cães criados em apartamentos. Passear com seus animais é um hábito saudável, mas nem sempre é acompanhado do bom hábito de recolhimento das fezes emitidas pelos animais durante o passeio, que permanecem nas calçadas e expõem a população circulante aos mais variados riscos, que vão desde escorregões e traumatismos até infecções por agentes danosos à saúde humana.

Dados de prevalência de agentes parasitários encontrados em fezes de cães recolhidas nas vias públicas são úteis como indicadores de risco para a população animal e humana, bem como para campanhas de conscientização com enfoque em Saúde Pública.

O objetivo deste trabalho foi determinar a prevalência de parasitos em massas fecais recolhidas em vias públicas do município de Niterói, RJ.

\section{Material e métodos}

Foram realizados exames parasitológicos em amostras fecais encontradas em calçadas de bairros do município de Niterói, como parte integrante das atividades do Projeto de Extensão Cata-caca: campanha para a coleta de fezes em calçadas e praças públicas do município de Niterói, RJ. Este projeto tem como objetivo conscientizar proprietários de cães sobre a importância de manter áreas públicas limpas.

O município de Niterói está localizado na região metropolitana do Rio de Janeiro, possui uma população estimada de 491.907 habitantes e uma área territorial de 133.916km² (IBGE, 2012).

Foram recolhidas 240 amostras de massas fecais em calçadas de oito bairros do município de Niterói: Icaraí, Ingá, Santa Rosa, Vital Brazil, São Francisco, Charitas, Jurujuba e Piratininga. Utilizou-se número de 30 amostras por bairro. As coletas foram realizadas no período matutino, entre 8 e 9 horas da manhã, horário que rotineiramente os proprietários passeiam com seus cães.

Amostras da superfície das massas fecais foram coletadas e acondicionadas em frascos plásticos com tampa e estes em recipiente isotérmico sob refrigeração para envio ao Laboratório de Doenças Parasitárias da Faculdade de Veterinária da UFF. As amostras fecais foram processadas pelos métodos de centrífugoflutuação em solução de sulfato de zinco 33\% (Faust et al., 1938) e sedimentação simples (Hoffman et al., 1934), com leitura de três lâminas para cada amostra, sem morfometria.

Os dados obtidos foram analisados por cálculo de prevalência das massas fecais positivas e dos patógenos encontrados. $\mathrm{O}$ termo prevalência é utilizado segundo Bush et al. (1997).

\section{Resultados}

Os exames coproparasitológicos realizados revelaram a prevalência de 18,3\% (44/240) das massas fecais positivas para a presença de formas evolutivas de parasitos, sendo destas, 22,7\% (10/44) em Charitas, 20,4\% (9/44) em Jurujuba, 13,6\% (6/44) no Vital Brazil, 13,6\% (6/44) no Ingá e 11,4\% (5/44) em São Francisco, 9,1\% (4/44) em Santa Rosa, 4,5\% (2/44) em Icaraí e 4,5\% (2/44) em Piratininga.
Das formas evolutivas de parasitos encontradas, $76 \%$ foram ovos de ancilostomatídeos, $9 \%$ cápsulas ovígeras de Dipylidium sp., $9 \%$ ovos de Trichuris sp., $4 \%$ ovos de Toxocara sp. e 2\% formas vegetativas de Entamoeba sp. Estas últimas foram encontradas em processamento da técnica de sedimentação simples.

\section{Discussão}

Estudos sobre a presença de parasitos em amostras fecais de cães e gatos foram realizados por Serra et al. (2003), Brener et al. (2005), Santos et al. (2007), Katagiri e Oliveira-Sequeira (2008) e Miranda et al. (2008), revelando também uma alta prevalência de ovos de ancilostomatídeos. Não há relato de estudos coproparasitológicos anteriores, utilizando massas fecais encontradas em ambientes públicos para o município de Niterói. No entanto Coelho et al. (2011), em estudo similar a este, investigaram especificamente a presença de ovos de ancilostomatídeos em fezes coletadas de locais públicos no município de Andradina, SP, encontrando $64,3 \%$ das massas fecais positivas. A alta prevalência de massas fecais com ovos de ancilostomatídeos, é utilizada por Coelho et al. (2011) como parâmetro para a indicação de tratamento anti-helmíntico dos animais e controle de animais errantes na área de estudo.

Para a área estudada neste trabalho, são raros os animais errantes. No entanto, foi observada uma grande circulação de proprietários passeando com seus cães, inclusive sem o devido recolhimento das fezes, sugerindo que a maioria das massas fecais encontrada deveria pertencer a cães domiciliados. Os resultados indicam falta de preocupação de alguns proprietários com a limpeza urbana e também com a saúde de seus animais.

Katagiri e Oliveira-Sequeira (2008), ao realizarem estudo na cidade de Botucatu (São Paulo), utilizando questionários com donos de cães, verificaram que a maioria respondeu que não conhecia os parasitos intestinais de cães, os mecanismos de transmissão, os fatores de risco para infecções zoonóticas e as medidas específicas de profilaxia. Estes autores salientaram ainda que o risco de infecções zoonóticas por parasitos intestinais caninos pode ser alto, mesmo em uma das regiões mais desenvolvidas do Brasil.

\section{Conclusão}

A prevalência alta de ancilostomatídeos e a presença de outros agentes parasitários nas amostras fecais examinadas neste trabalho revelam que existe contaminação ambiental na área estudada, sugerindo risco à população humana. Medidas simples como o recolhimento das fezes dos animais, com destino adequado e o tratamento dos animais parasitados, são ações necessárias para evitar a transmissão de destes agentes parasitários.

Há necessidade de maior investimento público em campanhas educativas, visando uma mudança do comportamento de seus habitantes, atentando para questões relativas à posse responsável de animais domésticos, que incluem medidas preventivas de transmissão de zoonoses parasitárias. 


\section{Referências}

ACHA, P. SZYFRES B. 2003. Zoonosis y Enfermedades Transmisibles comunes al hombre y a los animales. Tercera Edición. OPS. Washington USA. 413 p.

BRENER, B., LISBOA, L., MATTOS, D. P. B. G., ARASHIRO, E. K. N., MILLAR, P.R., SUDRÉ, A. P., DUQUE, V. Frequência de enteroparasitas em amostras fecais de cães e gatos dos municípios do Rio de Janeiro e Niterói. R. Bras. Ci. Vet., v. 12, n. 12, n. 1/3, p. 102-105, jan./dez. 2005.

BUSH, A.O.; LAFFERTY, K.D.; LOTZ, J.M.; SHOSTAK, A.W. Parasitology meets ecology on its own terms: Margolis et al. revisited. J. Parasitol., v. 83, p. 575-583, 1997.

COELHO, W.M.; AMARANTE, A.F.; APOLINÁRIO,J de C; COELHO, N.M.; BRESCIANI, KD. Ocurrence of Ancylostoma in dogs, cats and public places from Andradina city, São Paulo state, Brazil. Rev.Inst.Med Trop.São Paulo, v .53, n. 4, p. 181-184, julaug. 2011

FAUST, E. C.; D'ANTONI, J. S.; ODOM, V.; MILLER, M. J.; PERES, C.; SAWITZ, W.; THOMEN, L. F; TOBIE, J. ; WALKER, J. H. A critical study of clinical laboratory thecnics for the diagnosis of protozoan cysts and helminth eggs in feces. I. Preliminary communication. Amer. J. Trop. Med., v. 18, p. 169-183, 1938.
HOFFMAN, W. A.; PONS, J. A., JANER, J. L. The sedimentation method in schistosomiasis mansoni. The Puerto Rico $\mathrm{J}$ Publi Health Trop. Med., v. 9, n. 3, p. 283-291. 1934.

IBGE. Instituto Brasileiro de Geografia e Estatística. Disponível em: http://www.ibge.gov.br/cidadesat/topwindow.htm?1 Acesso em 15 de junho de 2012.

KATAGIRI, S; OLIVEIRA-SEQUEIRA, TC. Prevalence of dog intestinal parasites and risk perception of zoonotic infection by dog owners in São Paulo State, Brazil. Zoonosis Public Health v. 55, n. 8-10, p. 406-413, oct. 2008.

MIRANDA, F. J. B., ALBERNAZ, A. P., GOMES, F. F., GARCIA, L. N. N. Parasitismo por Ancilostomatídeos em cães (Canis familiaris) domiliados em Campos dos Goytacazes, RJ. Ciência Animal Brasileira, v. 9, n. 4, p. 1042-1045, 2008.

SANTOS, F. A. G.; Yamammura, M. H.; VIDOTTO, O.; CAMARGO, P. L. 2007. Ocorrência de parasitos gastrointestinais em cães (Canis familiaris) com diarreia aguda oriundos da região metropolitana de Londrina, estado do Paraná, Brasil. Ciências Agrárias, v. 28, p. 257-268. 2007.

SERRA, C. M. B.; UCHOA, C. M. A.; COIMBRA, R. A. Exame parasitológico de fezes de gatos (Felis catus domesticus) from the metropolitan área of Rio de Janeiro, Brazil. Rev. Soc. Bras. Med Trop.,v. 36, n. 3, p. 331-334, maio-jun. 2003 